\title{
Extramedullary hematopoiesis associated with myeloproliferative neoplasm manifesting as pleural effusion: case report and review of literature
}

\author{
Rebecca Sonu • Joo Y. Song $\cdot$ Mingyi Chen
}

Received: 14 March 2012 / Accepted: 3 May 2012 /Published online: 24 May 2012

(C) Springer-Verlag 2012

\begin{abstract}
Extramedullary hematopoiesis (EMH) is a pathologic process associated with either a neoplastic myeloproliferation or a reactive hematological disorder. EMH most commonly occurs in the spleen and liver. Pleural EMH is rare and usually asymptomatic. We describe a 72-year-old man who presented with massive splenomegaly and a left pleural effusion. Pleural biopsy demonstrated EMH with an increase in CD34+ immature myeloid cells. The fluorescence in situ hybridization study performed on the myeloid cells in the pleural lesion confirmed clonal EMH with trisomy 8. The bone marrow biopsy revealed marked granulocytic and megakaryocytic hyperplasia associated with diffuse myelofibrosis. The cytogenetic study of the bone marrow showed trisomy 8 . No $B C R-A B L 1$ fusion, $J A K 2^{\mathrm{V} 617 \mathrm{~F}}$ or $M P L^{\mathrm{W} 515 \mathrm{~K} / \mathrm{L}}$ mutations were detected. Histological examination of the enlarged spleen showed extensive EMH associated with fibrosis. The final diagnosis of myeloproliferative neoplasm, favoring primary myelofibrosis, was given. A literature review of EMH in association with MPN is discussed.
\end{abstract}

Keywords Myelodysplastic/myeloproliferative neoplasm • Extramedullary hematopoiesis and pleural effusion

\section{Introduction}

Extramedullary hematopoiesis (EMH) is characterized by the formation of blood cells outside the bone marrow. It

R. Sonu $\cdot$ J. Y. Song $\cdot$ M. Chen $(\bowtie)$

Department of Pathology and Laboratory Medicine,

University of California, Davis, Medical Center,

PATH Bldg. 4400 V Street,

Sacramento, CA 95817, USA

e-mail: mingyi.chen@ucdmc.ucdavis.edu

J. Y. Song

e-mail: joo.song@ucdmc.ucdavis.edu occurs either as a manifestation of a systemic neoplastic myeloproliferative disorder or as a compensatory mechanism for marrow replacement by a fibrotic process. EMH may also occur in benign hematological conditions such as hemoglobinopathies or in hemolytic anemias where there is compensation for "ineffective erythropoietic" states.

Among the different subtypes of chronic myeloproliferative neoplasm (MPN), primary myelofibrosis (PMF) is most commonly associated with EMH. PMF is also known as "myelofibrosis with myeloid metaplasia," "agnogenic myeloid metaplasia," or "chronic idiopathic myelofibrosis." According to the 2008 World Health Organization [1] criteria for classification and diagnosis of myeloproliferative neoplasms, PMF is characterized as a prominent megakaryocytic and granulocytic proliferation with atypia accompanied by reticulin and/or collagen fibrosis. PMF is now known to constitute a clonal stem cell disease with variable recurrent molecular markers (e.g., JAK2 $2^{\mathrm{V} 617 \mathrm{~F}}, M P L^{\mathrm{W} 515 \mathrm{~L} / \mathrm{K}}$ ) and/or cytogenetic markers (e.g., del(13q), del(20q), +8, +9, and abnormalities involving chromosome 1, 7, and 12). Other myeloproliferative neoplasms that can also progress to myelofibrosis include polycythemia vera (PV) and essential thrombocythemia (ET), resulting in post-polycythemia vera MF and post-essential thrombocythemia MF, respectively.

We present a patient who presented with massive splenomegaly and pleural effusion. The pleural biopsy and splenectomy confirmed marked EMH in the setting of a myeloproliferative disease.

\section{Case history}

The patient is a 72-year-old male who first presented with splenomegaly, abdominal pain, and weight loss. He was treated with hydroxyurea. 
The peripheral blood showed pancytopenia (Fig. 1a). The bone marrow biopsy showed a markedly hypercellular marrow $(90 \%$ cellularity) with prominent granulocytic and megakaryocytic hyperplasia and dysplasia associated with diffuse reticulin and collagen fibrosis (MF-3) and mild osteosclerosis (Fig. 1b, c). The dysplastic megakaryocytes were clustered in a sinusoidal pattern along the increased reticulin fibers and had hyperchromatic lobulated "cloud like" nuclei with abnormal nuclear to cytoplasmic ratio (Fig. 1d). Immunohistochemical stains for CD34 and CD117 showed no increase in immature myeloid precursors or blasts (1-2\%). Cytogenetic studies of the bone marrow cells revealed $47, \mathrm{XY},+8[20]$. No $J A K 2^{\mathrm{V} 617 \mathrm{~F}}$ and $M P L^{\mathrm{W} 5 \mathrm{I} 5 \mathrm{~K} / \mathrm{L}}$ mutations were detected by molecular studies.

An ultrasound of the abdomen revealed moderate hepatomegaly and marked splenomegaly. A splenectomy was performed yielding a massively enlarged 3,150-gram $(28.0 \times 22.0 \times 9.5 \mathrm{~cm})$ spleen (Fig. 2a). Microscopic examination demonstrated diffuse EMH (Fig. 2b) with many erythroid islands and dysplastic megakaryocytes (Fig. 2c) in a background of reticulin fibrosis (Fig. 2d). The final diagnosis of splenic EMH with an underlying MPN favoring PMF was given.

Three months later, the patient complained of severe shortness of breath for 1 week. Upon admission, he was mildly pancytopenic with a decreased white blood cell count of $3.4 \mathrm{~K} / \mathrm{MM}^{3}$, a normocytic anemia with a hemoglobin of $8.2 \mathrm{gm} / \mathrm{dl}$, hematocrit of $26.5 \%$, and a decreased platelet count of $100 \mathrm{~K} / \mathrm{MM}^{3}$.

A chest radiograph showed a marked left pleural effusion (Fig. 3a). A subsequent video-assisted thoracoscopic surgical decortication revealed fibrinous pleural thickening and a massive pleural effusion consisting of approximately 2 liters of serosanguinous fluid.

Microscopically, the parietal pleural biopsy (Fig. 3b) showed a myeloid predominant EMH with marked MPO positivity (Fig. 3c) and increased CD34+ immature myeloid cells (Fig. 3d). The dysplastic megakaryocytes were positive for von Willebrand factor (Fig. 3e), and a few were also positive for CD34. Calretinin stain highlighted the scattered residual mesothelial cells along the pleural surface of the biopsy (Fig. 3f). Fluorescence in situ hybridization (FISH) performed on the myeloid cells in the pleural biopsy detected trisomy 8 in 42 out of 100 interphase cells (Fig. 4).

\section{Methods}

The hematoxylin and eosin (H\&E) and Wright-Giemsastained slides were reviewed. Immunohistochemical studies of the spleen and pleura were performed on formalin-fixed paraffin-embedded tissue using an automated immunostainer (Ventana Medical Systems, Tucson, AZ, USA) with antibodies to CD34, CD43, CD117, Ki-67, AE1/AE3, myeloperoxidase (MPO), hemoglobin A, and von Willebrand factor stains. Flow cytometry studies were performed as part of the clinical workup of the bone marrow. The medical records were reviewed for clinical presentation, staging, laboratory data, radiological studies, treatment, and outcome.

$J A K 2^{\mathrm{V} 617 \mathrm{~F}}$ mutation was performed on the bone marrow aspirate cells. DNA was isolated and subjected to allele-specific PCR amplification. The reaction used an oligonucleotide primer set specific for the exon 14 of $J A K 2$ on chromosome 9 and an
Fig. 1 a Peripheral blood smear showing pancytopenia (original magnification, $\times 200$ ). b Low-power, bone marrow biopsy showing marked hypercellularity with granulocytic and megakaryocytic hyperplasia (original magnification, $\times 40$ ). c Bone marrow biopsy with reticulin myelofibrosis and osteosclerosis (original magnification, $\times 100$ ). d High-power, bone marrow biopsy showing clusters of dysplastic megakaryocytes (original magnification, $\times 200$ )
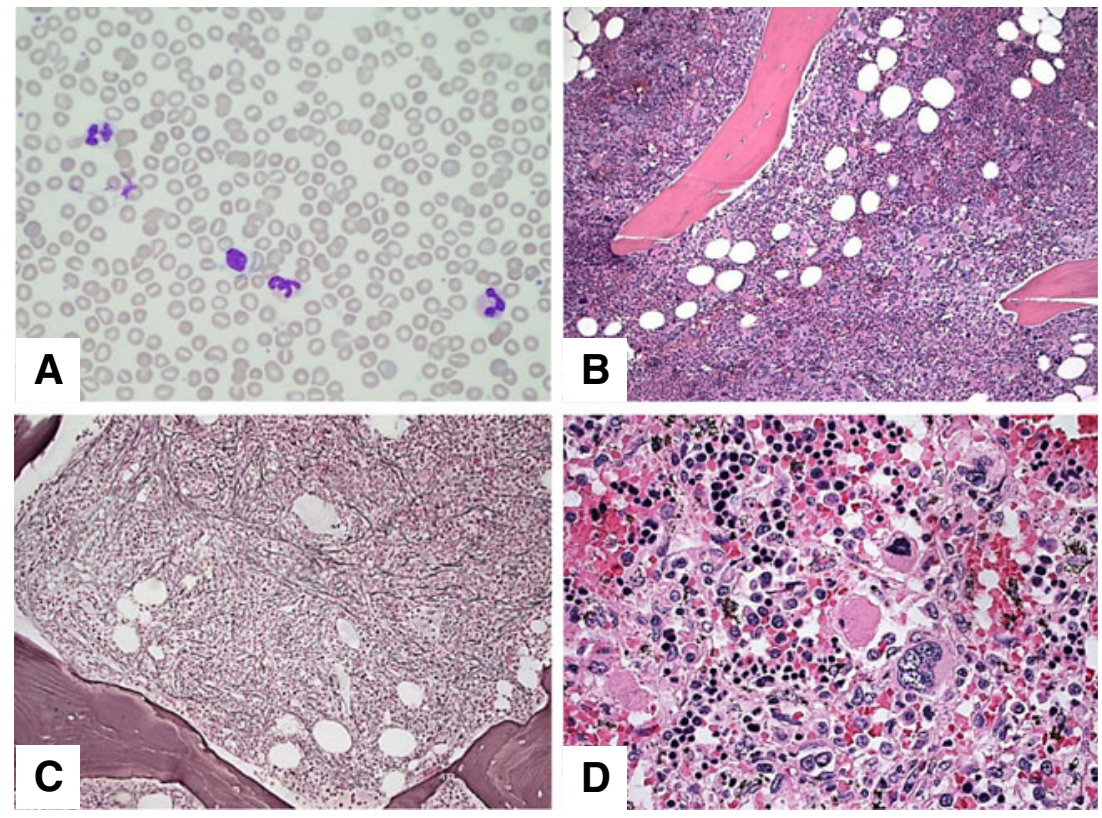
Fig. 2 a Gross picture of a cross-section of the enlarged spleen. b Low-power, H\&Estained section of spleen showing diffuse EMH (original magnification, $\times 100)$. c Highpower, EMH of the spleen with many erythroid islands and clusters of dysplastic megakaryocytes (original magnification, $\times 200$ ); a hyperchromatic

hyperlobated dysplastic megakaryocyte with a "cloud-like" nucleus. d Increased reticulin fibrosis associated with $\mathrm{EMH}$ (original magnification, $\times 100$ )
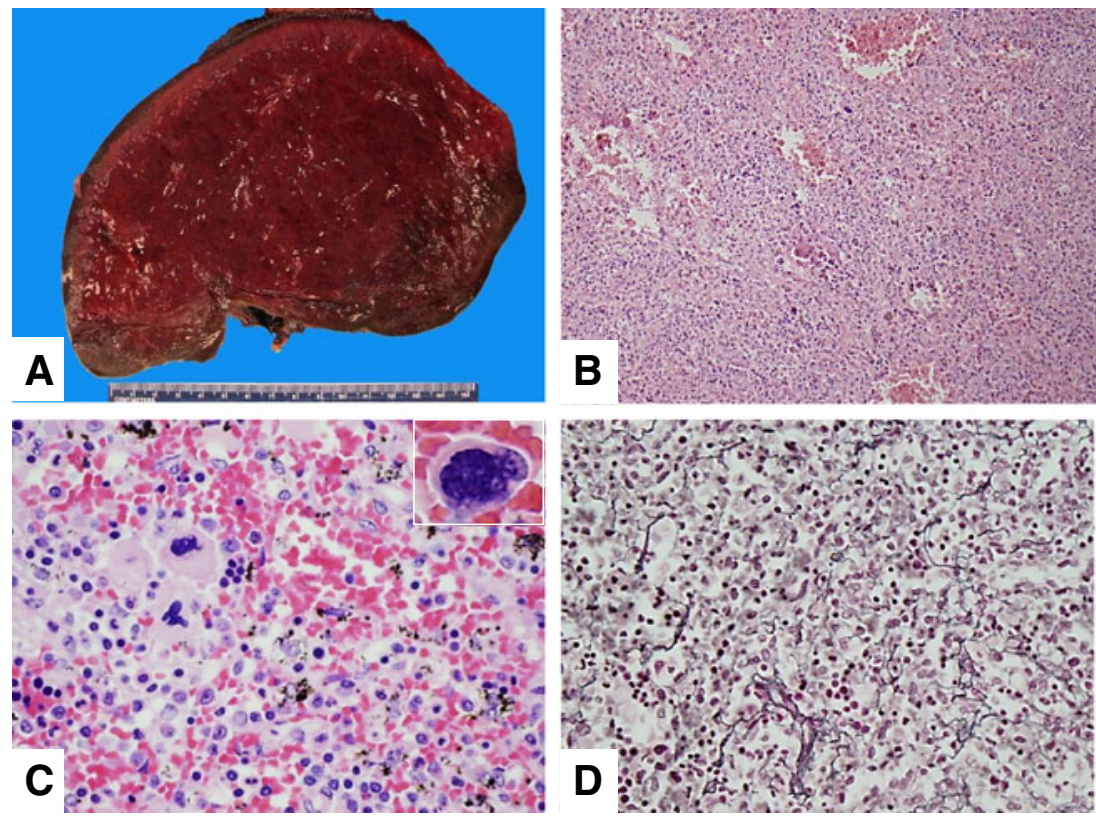

allele-specific primer that specifically initiated amplification from the allele containing the point mutation in codon 617 . MPL exon 10 mutation analysis was performed on peripheral blood cells. Genomic DNA was extracted, and Sanger sequencing was used to evaluate for mutations in $M P L$, exon 10.
Conventional chromosome analysis of the bone marrow was performed by the standard cytogenetics protocols for neoplastic studies. Twenty cells were available for analysis with a karyotype of 47, XY, +8. Interphase FISH analysis was performed on paraffin blocks and performed at Clarient Diagnostic Services
Fig. 3 a Chest radiograph showing a large left pleural effusion and loculation. b H\&E of the pleural biopsy showing myeloid predominant EMH (original magnification, $\times 200$ ) c EMH with sheets of MPO+ myeloid cells. d EMH with scattered CD34+ immature myeloid cells. e von Willebrand factor (vWF)-positive dysplastic megakaryocytes. f Calretinin positive mesothelial cells present along pleural surface $(\mathbf{c}-\mathbf{f}$, original magnification, $\times 100$ )
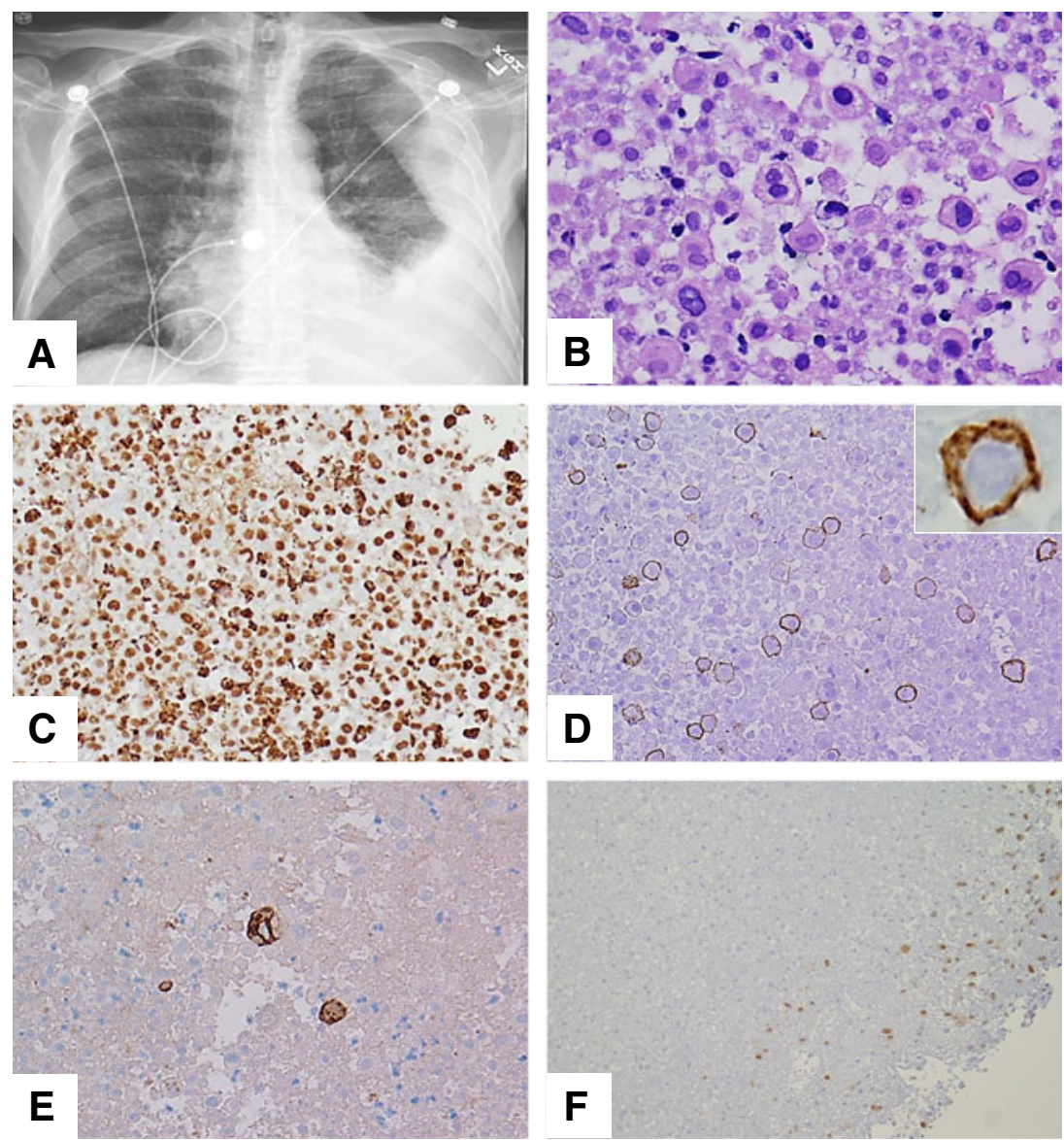


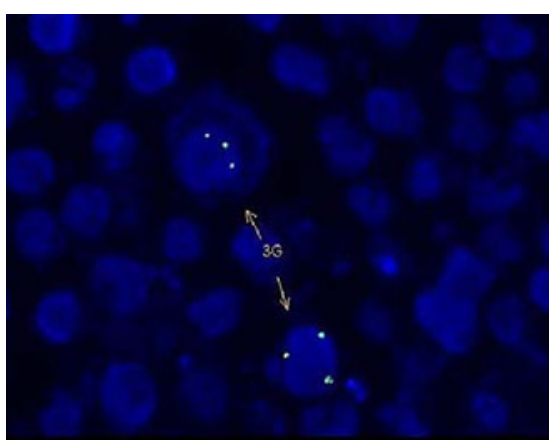

Fig. 4 FISH image detecting trisomy 8 in large immature myeloid cells in pleural EMH

(Aliso Viejo, CA) using the CEP8 probe to assess the pleural biopsy for the presence of trisomy 8 . Analysis of 100 interphase cells with each probe set showed an abnormal hybridization pattern; $42 \%$ of cells showed three copies of the CEP8 probe. The final result was nuc ish8p10(CEP8x3)[42/100], confirming the diagnosis of trisomy 8 in the immature myeloid cells.

\section{Discussion}

Extramedullary hematopoiesis

EMH is defined by the proliferation of marrow elements in extramedullary sites. In adults, EMH most commonly occurs in the spleen, as well as the liver and lymph nodes. EMH in other organs such as the fallopian tubes, ovaries [2], orbit, middle ear, thyroid, heart [3], and central nervous system [4] has been reported in the literature. EMH can occur in the setting of either a neoplastic myeloproliferation or in a reactive hematological disorder (Table 1).

In benign conditions, EMH may occur due to an underlying hematological disease or chronic intramedullary hemolytic disorder such as thalassemia or spherocytosis [3]. EMH is more commonly seen where ineffective hematopoiesis compounds the hemolytic process. In hemoglobinopathies, circulation of immature cells can be filtrated and trapped in the spleen or in other sites. These cells can then proliferate into hematopoietic elements due to "ineffective hematopoiesis" or fibrosis in the bone marrow as seen with osteosclerosis [3]. Eskazan and authors recently showed that, in a patient with beta-thalassemia, compensatory intracranial EMH can be reversed with hypertransfusion [5].

EMH may also occur as a manifestation of a neoplastic myeloproliferative disorder. Also known as myeloid metaplasia, one theory suggested that EMH is a consequence of sequestration, accumulation, and proliferation of circulating clonal myeloid progenitors [6]. In some myeloproliferative disorders, transformed stem cells may circulate outside of the bone marrow to seed hematopoiesis at atypical sites.

Extramedullary hematopoietic involvement in the mediastinum and pleura is a rare phenomenon. In the current

Table 1 Benign versus clonal: extramedullary hematopoiesis of the spleen

\begin{tabular}{|c|c|c|c|}
\hline & Etiology & EMH histology & Immunohistochemistry/other \\
\hline \multirow[t]{11}{*}{ Benign } & Erythroid disorders & $\begin{array}{l}\text { Trilineal with erythroid predominance } \\
\text { and occasional megakaryocytes }\end{array}$ & Lack of CD34+ cells \\
\hline & Thalessemias & & \\
\hline & $\begin{array}{l}\text { Hereditary spherocytosis } \\
\text { Pyruyate kinase deficiency }\end{array}$ & & \\
\hline & $\begin{array}{l}\text { Pyruvate kinase deficiency } \\
\text { Autoimmune }\end{array}$ & & \\
\hline & AIHA & & \\
\hline & ITP & & \\
\hline & TTP-HUS & & \\
\hline & Reactive & & \\
\hline & $\begin{array}{l}\text { Post-BMT, trauma, ITP } \\
\text { SCID }\end{array}$ & & \\
\hline & Bone metastasis (eg. carcinoma) & Trilineal EMH without atypia, & \\
\hline & Cytokine induced (eg. G-CSF) & Myeloid predominant & \\
\hline \multirow[t]{7}{*}{ Clonal } & Myeloproliferative disorders & $\begin{array}{l}\text { Predominantly unilineal or trilineal } \\
\text { with atypical megakaryocytes }\end{array}$ & Increased CD34+, CD117+ \\
\hline & & EMH with sclerotic stroma (SEMHT) & \\
\hline & CIMF & $\begin{array}{l}\text { (Splenic EMH patterns: diffuse, } \\
\text { nodular, immature granulocyte } \\
\text { predominant microinfarction) }\end{array}$ & $\begin{array}{l}\text { Dysplastic megakaryocytes CD42b+, } \\
\text { CD34+ (hyperchromatic, hyper/ } \\
\text { hypolobated in clusters) }\end{array}$ \\
\hline & CML, PV, ET & & \\
\hline & MDS/MPN & Features of both MDS and MPN & Dysplastic erythroids (multinuclear, \\
\hline & $\underline{\text { MDS }}$ & $\begin{array}{l}\text { Predominantly unilineal or trilineal } \\
\text { With increased monocytes/macrophages, } \\
\text { dysplastic megakaryocytes }\end{array}$ & Aberrant cytogenetics or FISH studies \\
\hline & $\frac{\text { Others }}{\text { AML }}$, mastocytosis, lymphoma & & \\
\hline
\end{tabular}

AIHA autoimmune hemolytic anemia, $A M L$ acute myelogenous leukemia, $B M T$ bone marrow transplant, $C D$ cluster of differentiation, $C I M F$ chronic idiopathic myelofibrosis, $C M L$ chronic myelogenous leukemia, $C M M L$ chronic myelomonocytic leukemia, $E M H$ extramedullary hematopoeisis, ET essential thrombocythemia, G-CSF granulocyte-colony stimulating factor, ITP idiopathic thrombocytopenia, JMML juvenile myelomonocytic leukemia, $M D S$ myelodysplastic syndrome, $P V$ polycythemia vera, $S C I D$ severe combined immunodeficiency, $S E M H T$ sclerosing extramedullary hematopoietic tumor [27], TTP-HUS thrombotic thrombocytopenic purpura-hemolytic uremic syndrome 
case, the demonstration of trisomy 8 in the pleural biopsy confirmed the clonal neoplastic nature of the pleural EMH. Furthermore, the presentation of EMH in this case is consistent with an underlying malignant condition. It has been suggested that both circulating progenitor cell trapping and abnormal cytokine stimulation of embryonic hematopoietic sites are implicated mechanisms of hepatosplenic EMH in PMF. Such a contention is supported by the high concordance between cytogenetic findings in the bone marrow and pleural tissue in PMF.

The clonal EMH cells of neoplastic/malignant EMH are morphologically, immunohistochemically, and molecularly distinct from normal hematopoietic cells [7]. Clinically, these cells are systemically involved. Morphologically, malignant EMH cells are often unilineage (myeloid in predominance) with some dysplastic features. The neoplastic clonality can be detected by aberrant cytogenetics or FISH studies (Table 1). Detection of immature cells with positive CD34 and CD117 immunostains on concurrent blood and bone marrow findings are imperative in determining acute leukemic transformation [7].

\section{EMH and primary myelofibrosis}

Chronic myeloproliferative neoplasms, especially PMF, are most commonly associated with EMH in the spleen. In splenic $\mathrm{EMH}$, the red pulp is often expanded with erythroid, granulocytic, and megakaryocytic lineages. Patients with symptomatic splenomegaly usually undergo palliative splenectomies, which often results in EMH in uncommon sites without hematopoietic capacity, such as the bladder [8], skin [9], and pleura [10]. However, several autopsy cases with clonal EMH arising in areas such as the thoracic spine, dura, liver, and kidneys have occurred in cases in which there has not been a prior splenectomy [4], suggesting that the spleen is not always the first or only site of EMH. With an underlying MPN, the myeloid lineage may present as the predominant cell type and prominent fibrosis may be present in the involved organs.

Several theories have been postulated to explain the pathogenesis of EMH in PMF. EMH in myeloproliferative disorders was originally believed to arise from the reactivation of fetal hematopoietic elements [11]. Due to the bone marrow fibrosis, EMH has been postulated to occur as a compensatory mechanism for inadequate hematopoiesis [3], as similarly described in "benign" EMH. Current consensus suggests that EMH is a consequence of sequestration, accumulation, and proliferation of circulating progenitors cells in extramedullary sites [6].

\section{JAK2 mutation in EMH}

Several molecular studies have demonstrated that EMH in PMF is actually due to a leukemic infiltrate residing in extramedullary organs. In patients with PMF, concordant $J A K 2^{\mathrm{V} 617 \mathrm{~F}}$ mutations in the bone marrow and EMH cells of the skin [9] and spleen [6,12] have demonstrated that EMH cells are actually derived from the bone marrow as part of a neoplastic clonal process [13].

The JAK2 mutation, more commonly the $J A K 2^{\mathrm{V} 617 \mathrm{~F}}$, is strongly associated with MPNs. The mutation occurs due to the substitution of valine to phenylalanine at the amino acid 617. The Janus tyrosine kinase proteins are instrumental in mediating signal transduction pathways associated with cytokine receptors and cell proliferation [14]. Dysregulated signaling is a hallmark of MPNs as evidenced by the identification of the activating $J A K 2^{\mathrm{V} 617 \mathrm{~F}}$ somatic mutation. Approximately $50 \%$ of cases of PMF, $98 \%$ of polycythemia vera and $50 \%$ of essential thrombocythemia are associated with this mutation [14]. Currently, the $J A K 2^{\mathrm{V} 617 \mathrm{~F}}$ mutation has been useful as a molecular marker in determining clonality of EMH in patients with MPNs.

However, in $J A K 2^{\mathrm{V} 617 \mathrm{~F}}$-negative patients, the presence of a $J A K 2$ exon 12 mutation also meets the WHO criterion for establishing clonality. Exon 12 mutations have been found in patients with PV who present with erythrocytosis but are typically not associated with ET or PMF. Because a significant proportion of patients with ET or PMF are negative for $J A K 2$ mutations, other somatic mutations have been sought; hence mutations in the myeloproliferative leukemia gene $(M P L)$ were identified. $M P L$, found at chromosome $1 \mathrm{p} 34$, encodes the thrombopoietin receptor that works in concert with thrombopoietin for platelet production. Acquired MPL mutations (W515L and W515K) are associated with severe anemia and have been detected in patients with ET or PMF but not in patients with PV. Besides JAK2, the development of MPNs also can be caused by other mutations, including $L N K, T E T 2, I D H 1, I D H 2, C B L, E Z H 2$, ASXL1, and DNMT3A [15].

The detection of concurrent cytogenetic abnormalities between the bone marrow and EMH in $J A K 2^{\mathrm{V} 617 \mathrm{~F}}$-negative, $B C R-A B L 1$-negative patients reinforces the idea that EMH is "neoplastic" in nature. In this case, the concurrent trisomy 8 detected in the bone marrow and pleural EMH shows that the $J A K 2^{\mathrm{V} 617 \mathrm{~F}}$ mutation is not the only aberration involved in the malignant nature of EMH in PMF. In addition, loss of heterozygosity studies and X-chromosome inactivation analysis have also shown that clonal abnormalities are frequently present in spleens with neoplastic myeloid proliferations [16].

While peripheral blood contamination in the pleural biopsy should be considered as a possibility for the concurrent trisomy 8 at both sites, ancillary studies have ruled out contamination. In the FISH study, the demonstration of trisomy 8 in the large immature cells/blasts confirmed the clonal neoplastic nature of the pleural EMH (Fig. 4). In this case, no increase of immature myeloid cells was detected in 
the peripheral blood. By immunohistochemistry, there were sheets of MPO-positive myeloid cells and an increase in CD117+/CD34+ myeloblasts. The dysplastic morphology of the megakaryocytes also provided additional evidence.

Differential diagnosis: myelodysplastic/myeloproliferative neoplasms (MDS/MPN)

This case also featured overlapping dysplastic and proliferative features, such as dysplastic megakaryocytes, marked marrow fibrosis, and organomegaly, in which a myelodysplastic/myeloproliferative neoplasm (MDS/MPN) should be considered in the differential. The negative JAK2 mutation and negative $B C R-A B L$ is consistent with the 'unclassifiable' subtype of MDS/MPN.

MDS/MPNs are clonal myeloid neoplasms characterized at the time of initial presentation by the simultaneous presence of both myelodysplastic and myeloproliferative features [17]. According to the World Health Organization 2008 classification for hematopoietic and lymphoid tissues, the four subtypes of MDS/MPNs include chronic myelomonocytic leukemia (CMML), juvenile myelomonocytic leukemia (JMML), atypical chronic myeloid leukemia BCRABL1-negative, MDS/MPN-unclassifiable (MDS/MPNUC), along with a provisional category, refractory anemia with ringed sideroblasts and thrombocytosis [1]. However, due to the absence of distinctive genetic and biologic features for MDS/MPN, a comprehensive clinical and laboratory assessment with careful integration of the morphological, immunophenotypic, genetic, and clinical characteristics is required for diagnosis.

MDS/MPN-UC is a diagnosis of exclusion. The disease presents initially with hybrid dysplastic or proliferative features. The complete blood cell count should demonstrate cytopenias and/or cytoses. Existing blast equivalents should be delineated from promonocytes to distinguish from CMML, JMML, and AML. Blasts and/or blast equivalents should number $<20 \%$ in the peripheral blood and bone marrow. A positive $J A K 2$ mutation should be indicative of a myeloproliferative neoplasm. Finally, genetic testing must be performed to exclude $B C R / A B L 1$ related neoplasms, $P D G F R A / B, F G F R 1$, isolated del5q, and low blast count AML defining translocations [18].

MDS/MPN-UC is a very rare disease. EMH in CMML and JMML have been reported [7], although not in MDS/ MPN-UC. More studies are needed to further define the clinical presentation, risk stratification, and prognostic factors of the disease.

\section{Trisomy 8}

The significance of trisomy 8 in EMH and PMF is unknown. Associated and often compound cytogenetic abnormalities are also seen in about $30 \%$ of PMF patients. The most frequent abnormalities included 20q-, 13q- $,+8,+9$, $12 \mathrm{p}$-, and abnormalities of chromosomes 1 , isochromosome $17 \mathrm{q}$ and 7 [6]. Trisomy 8 and 12p-, in addition to advanced age and anemia, were independent prognostic determinants of inferior survival [19]. In addition, trisomy 8 or amplification of 8q24 (MYC) was found to be almost exclusively detected in $J A K 2^{\mathrm{V} 617 \mathrm{~F}}$ negative cases with a myeloproliferative-blast phase [20].

\section{Transformation}

In this case, the pleural biopsy showed increased CD34+/ CD117+ positive immature myeloid cells indicating a possible early phase of transformation to acute leukemia. It has been reported that the blast phase in PMF may first occur in immature myeloid precursors in extramedullary sites [21]. In the pleura, leukemic transformation has been reported to occur in $10 \%$ to $20 \%$ of patients with myeloid predominant EMH [22]. In addition, the diffuse pattern of splenic EMH has been reported to confer a higher incidence of histopathologic transformation associated with a poorer prognosis compared with a nodular pattern [6].

\section{Therapeutic targets in MPN}

Hydroxyurea has been described as a possible treatment for EMH in chronic myeloid proliferative disorders. In the case of intrathoracic extramedullary hematopoiesis with effusion, it is not known whether hydroxyurea is effective. In our case, the patient was already being treated with hydroxyurea for 3 years before he presented with pleural effusion. Therefore, it appears that the hydroxyurea did not prevent the progression of the underlying MPN neoplastic process and the complication of pleural involvement by EMH. For nonhepatosplenic EMH, the radiosensitivity of EMH to lowdose radiotherapy has been shown to be as efficacious [23].

Since aberrant $J A K 2$ activation is closely related to MPNs, multiple $J A K 2$ inhibitors have been applied in clinical trials, and the most recent data showed promising results for treating patients with PMF, PV, and ET. Furthermore, other investigational non-tyrosine kinase inhibitor approaches such as immunomodulatory agents and pegylated interferon- $\alpha$ are under investigation. Recent studies have emphasized the importance of epigenetic modifications in the pathogenesis of MPNs. In vitro experiments with DNA methyl-transferase and histone deacetylase inhibitors on patient samples provide promising results, and clinical trials for the treatments of PMF patients are under way [24-26]. 


\section{Conclusion}

The pathogenesis of EMH may stem from either a benign or malignant underlying hematological condition. In the case of neoplastic/clonal EMH, myeloid metaplasia can arise from multipotential stem cells in any mesenchymal tissue resulting in a myeloid predominant EMH. Pleural EMH can become clinically important both symptomatically and from the standpoint of the differential diagnosis. Therefore, in a patient with a predisposing hematological condition, EMH should be considered in a patient with an unexplained cause of pleural effusion. The presence of immature myeloid cells in a pleural lesion may indicate an early developing focus of transforming acute leukemic cells.

Acknowledgement We thank Drs. Ralph Green and John Bishop for the critical reading of this manuscript.

Conflict of interest The authors declare that they have no conflict of interest.

\section{References}

1. Swerdlow SH, Campo E, Harris NL, Jaffe ES, Pileri SA, Stein H, Thiele J, Vardiman JW (eds) (2008) World Health Organization classification of tumours of haematopoietic and lymphoid tissues. IARC

2. Rabischong B, Larrain D, Charpy C, Dechelotte PJ, Mage G (2010) Extramedullary hematopoiesis and myeloid metaplasia of the ovaries and tubes in a patient with myelofibrosis: case report and concise review of the reported cases. J Clin Oncol 28(28):e511-e512

3. O'Malley DP (2007) Benign extramedullary myeloid proliferations. Mod Pathol 20(4):405-415

4. Zherebitskiy V, Morales C, Del Bigio MR (2011) Extramedullary hematopoiesis involving the central nervous system and surrounding structures. Hum Pathol 42(10):1524-1530

5. Eskazan AE, Ar MC, Baslar Z (2012) Intracranial extramedullary hematopoiesis in patients with thalassemia: a case report and review of the literature. Transfusion doi:10.1111/j.1537-2995.2011.03499.x

6. Mesa RA, Li CY, Schroeder G, Tefferi A (2001) Clinical correlates of splenic histopathology and splenic karyotype in myelofibrosis with myeloid metaplasia. Blood 97(11):3665-3667

7. O'Malley DP, Kim YS, Perkins SL, Baldridge L, Juliar BE, Orazi A (2005) Morphologic and immunohistochemical evaluation of splenic hematopoietic proliferations in neoplastic and benign disorders. Mod Pathol 18(12):1550-1561

8. Willems SM, Beck J, von der Thusen J (2008) Macroscopic hematuria in patient with myelofibrosis caused by extramedullary hematopoiesis of bladder. Urology 72(4):e13-e14, 948

9. Fraga GR, Caughron SK (2010) Cutaneous myelofibrosis with $J A K 2^{\mathrm{V} 617 \mathrm{~F}}$ mutation: metastasis, not merely extramedullary hematopoiesis! Am J Dermatopathol 32:727-730
10. Schwarz C, Bittner R, Kirsch A et al (2009) A 62 year-old woman with bilateral pleural effusions and pulmonary infiltrates caused by extramedullary hematopoiesis. Respiration 78:110-113

11. Dameshek W (1951) Editorial: some speculations on the myeloproliferative syndromes. Blood 6:372-375

12. Hsieh PP, Olsen RJ, O'Malley DP et al (2007) The role of Janus Kinase $2 \mathrm{~V} 617 \mathrm{~F}$ mutation in extramedullary hematopoiesis of the spleen in neoplastic myeloid disorders. Mod Pathol 20:929-935

13. Konoplev S, Hsieh PP, Chang CC, Medeiros LJ, Lin P (2007) Janus kinase $2 \mathrm{~V} 17 \mathrm{~F}$ mutation is detectable in spleen of patients with chronic myeloproliferative diseases suggesting a malignant nature of splenic extramedullary hematopoiesis. Hum Pathol 38:1760-1763

14. Shih AH, Levine RL (2011) Molecular biology of myelodysplastic syndromes. Semin Oncol 38(5):613-620

15. Vannucchi AM, Biamonte F (2011) Epigenetics and mutations in chronic myeloproliferative neoplasms. Haematologica 96(10):1398 1402

16. O'Malley DP, Orazi A, Wang M, Cheng L (2005) Analysis of loss of heterozygosity and $\mathrm{X}$ chromosome inactivation in spleens with myeloproliferative disorders and acute myeloid leukemia. Mod Pathol 18:1562-1568

17. Orazi A, Germing U (2008) The myelodysplastic/myeloproliferative neoplasms: myeloproliferative neoplasms with dysplastic features. Leukemia 22:1308-1319

18. Foucar K (2009) Myelodysplastic/myeloproliferative neoplasms. AJCP 132:281-289

19. Tefferi A, Mesa RA, Schroeder G, Hanson CA, Li CY, Dewald GW (2001) Cytogenetic findings and their clinical relevance in myelofibrosis with myeloid metaplasia. Br J Haematol 113:763771

20. Thoennissen NH, Krug UO, Lee DH et al (2010) Prevalence and prognostic impact of allelic imbalances associated with leukemic transformation of Philadelphia chromosome-negative myeloproliferative neoplasms. Blood 115:2882-2890

21. Knoll B, Rea DJ, Szoztek J, Nagorney DS, Tefferi A, Mesa RA (2007) Images in hematology: extramedullary hematopoiesis. Am J Hematol 83(2):171

22. Nadrous HF, Krowka MJ, McClure R, Tefferi A, Lim KG (2004) Agnogenic myeloid metaplasia with pleural extramedullary leukemic transformation. Leuk Lymphoma 45(4):815-818

23. Tefferi A (2012) Primary myelofibrosis: 2012 update on diagnosis, risk stratification and management (2012). Am J Hematol 86 (12):1017-1026

24. Agrawal M, Garg RJ, Cortes J, Kantarjian H, Verstovsek S, Quintas-Cardama A (2011) Experimental therapeutics for patients with myeloproliferative neoplasias. Cancer 117(4):662-676

25. Tibes R, Mesa RA (2011) Myeloproliferative neoplasms 5 years after discovery of $J A K 2^{\mathrm{V} 617 \mathrm{~F}}$ : what is the impact of JAK2 inhibitor therapy? Leuk Lymphoma 52(7):1178-1187

26. Hemavathy K, Wang JC (2009) Epigenetic modifications: new therapeutic targets in primary myelofibrosis. Curr Stem Cell Res Ther 4(4):281-286

27. Gualco G, Ojopi EBP, Chioato L, Cordeiro DL, Negretti F, Bacchi CE (2010) Postsplenectomy sclerosing extramedullary hematopoietic tumor with unexpected good clinical evolution. Appl Immunohistochem Mol Morphol 18(3):291-295 